\title{
Análise Comparativa do Crescimento de Biótipos de Picão-Preto (Bidens pilosa) Resistente e Suscetível aos Herbicidas Inibidores da ALS ${ }^{1}$
}

\author{
Growth Analysis of Bidens pilosa Biotypes Resistant and Susceptible to Als Inhibitor Herbicides
}

\author{
CHRISTOFFOLETI, P.J. ${ }^{2}$
}

\begin{abstract}
RESUMO - A resistência de biótipos de plantas daninhas aos herbicidas inibidores da acetolactato sintase (ALS) é causada pela insensibilidade desta enzima aos herbicidas que inibem sua atividade catalítica. A insensibilidade da enzima é decorrente de uma alteração estrutural, resultado da substituição de certos aminoácidos no sítio de ação do herbicida. Esta alteração na enzima pode eventualmente resultar, além da resistência ao herbicida, em modificações na taxa de crescimento da planta, fato este comprovado para os biótipos resistentes aos herbicidas inibidores do fotossistema II, os quais apresentam taxa de crescimento prejudicada pela alteração no sítio de ação sofrida pelo herbicida. Esta possível diminuição na taxa de crescimento da planta resistente tem conseqüências diretas na competitividade do biótipo e, portanto, na sua dinâmica dentro da população, afetando diretamente as estratégias de manejo da resistência. A presente pesquisa foi desenvolvida com o objetivo de comparar a taxa de crescimento de dois biótipos da planta daninha picãopreto (Bidens pilosa), sendo um resistente e um suscetível aos herbicidas inibidores da ALS. Um experimento foi montado em casa de vegetação, em vasos com capacidade de $5 \mathrm{~L}$, sendo uma planta de cada biótipo por vaso, coletando-se a biomassa seca destas plantas e a área foliar semanalmente, iniciando-se 14 dias após o plantio. Os resultados de crescimento da biomassa e área foliar foram ajustados utilizando-se a função de Richards (log-logística). Desta análise, foram derivadas a taxa de crescimento absoluto (TCA), a taxa de crescimento relativo (TCR) e a taxa de assimilação fotossintética líquida (TAL). O biótipo suscetível apresentou peso de biomassa seca superior ao resistente nas primeiras fases do crescimento, porém no final do ciclo o biótipo resistente igualou-se em tamanho de área foliar, pois apresentou, principalmente no início do ciclo de crescimento, TCA, TCR e TAL maiores que o suscetivel. Dessa forma, concluiu-se que o biótipo de Bidens pilosa resistente aos herbicidas inibidores da ALS apresenta a mesma eficiência de produção de biomassa no final do ciclo. É provável que, quando em competição entre si e com as culturas, possua a mesma competitividade, sendo a dominância numérica de um biótipo sobre o outro decorrente apenas da pressão de seleção causada pelo herbicida.
\end{abstract}

Palavras-chave: resistência aos herbicidas, análise de crescimento, função de Richards, taxa de crescimento.

\begin{abstract}
The resistance of weed biotypes to acetolactate synthase (ALS) inhibitor herbicides is due to this enzyme's lack of sensitivity to ALS inhibitor herbicides, which inhibit its catalytic activity. ALS insensitivity results from a structural change in the aminoacid sequence, exactly in the site of action of these herbicides. Eventually this modification in the enzyme may result in a reduced plant growth rate. Such reduction was also observed in biotypes resistant to Photosystem II inhibitor herbicides. The possibility of a lower growth rate of the resistant plant may directly affect biotype competitiveness, its population dynamics and, as a consequence, resistance management strategies. The objective of this research was to compare the growth rates of both
\end{abstract}

Recebido para publicação em 22/1/2001 enviado para publicação em 27/3/2001.

2 Professor Associado do Departamento de Produção Vegetal da ESALQ/USP. Av. Pádua Dias, 11 - 13418-900 Piracicaba-SP, <pjchrist@ carpa.ciagri.usp.br> - Tel. (19) 429-4190. 
resistant and susceptible Bidens pilosa biotypes to ALS inhibitor herbicides. The experiment was conducted in a greenhouse, using one plant per pot of $5 \mathrm{~L}$ capacity. Four plants per biotype were harvested weekly, starting 14 days after planting, and the leaf area and dry biomass were measured. The Richards function fitted to the data enabled the derivation of absolute growth rate, relative growth rate and net assimilation rate. The susceptible biotype had a higher biomass accumulation during the early stages, with both biotypes having the same size, afterwards. The higher net assimilation rate of the resistant biotype during the early stages of growth was balanced by its lower size during the first four weeks of growth. It was concluded that both biotypes have the same size, being very likely that resistant and susceptible Bidens pilosa have the same competitiveness.

Key words: herbicide resistance, growth analysis, Richards function, growth rate.

\section{INTRODUÇÃO}

A utilização de modelos matemáticos para descrição de fenômenos que ocorrem na agricultura tem sido uma ferramenta de uso freqüente nas pesquisas agrícolas (France \& Thornley, 1984). Na literatura estão descritos modelos de previsão da taxa de evolução de resistência aos herbicidas em populações de plantas daninhas, e um dos parâmetros determinantes destes modelos é a adaptabilidade relativa dos biótipos resistentes (R) e suscetíveis (S). No modelo de previsão criado por Gressel \& Segel (1978), a adaptabilidade ecológica do biótipo R tem importância fundamental na taxa de evolução da resistência. Na ausência da pressão de seleção pelo herbicida, o biótipo de menor adaptabilidade ecológica permanece raro na população, podendo até ter sua freqüência diminuída. Dessa forma, nas recomendações das estratégias de prevenção e manejo da resistência de plantas daninhas aos herbicidas, é fundamental que sejam conhecidas a competitividade e adaptabilidade dos biótipos $\mathrm{R}$ e $\mathrm{S}$ de plantas daninhas.

A capacidade de sobrevivência e reprodução de um biótipo em uma população determina a sua adaptabilidade ecológica, a qual depende de características biológicas, como taxas de germinação, crescimento e produção de sementes. Maxwell et al. (1990) incluíram diversas características biológicas que influem na adaptabilidade de um biótipo $\mathrm{R}$ em um modelo de previsão de evolução da resistência. Para que as medidas de prevenção e manejo de resistência possam ser recomendadas de forma racional e com base em informações consistentes de previsão da evolução da resistência em populações de plantas daninhas, é necessário que o comportamento biológico dos biótipos suscetiveis e resistentes seja caracterizado (Gill et al., 1996).

Alguns trabalhos científicos têm mostrado diferentes taxas de germinação entre os biótipos R e S. O biótipo suscetível da planta daninha Brassica campestris germina 12 a 18 horas antes que o biótipo resistente aos herbicidas do grupo químico das triazinas (Mapplebeck et al., 1982). Dyer et al. (1993) determinaram que sementes de população de Kochia scoparia resistente a sulfoniluréias germinaram mais rápido que sementes de uma população suscetível a $4,6{ }^{\circ} \mathrm{C}$, mas não a $10,6^{\circ} \mathrm{C}$. Foi encontrado um nível elevado de valina, leucina e isoleucina livre na população resistente, em comparação com a população suscetível, sendo esta a possível causa da germinação mais rápida do biótipo R. Em estudos posteriores, Thompson et al. (1994) verificaram que a taxa de germinação diferencial entre os biótipos R e S de K. scoparia é dependente da temperatura, pois o biótipo $\mathrm{R}$ germina mais rápido a $8{ }^{\circ} \mathrm{C}$ que a $18{ }^{\circ} \mathrm{C}$, mas não a $28^{\circ} \mathrm{C}$.

A maioria dos estudos relatados na literatura sobre a comparação da competitividade entre os biótipos $\mathrm{R}$ e $\mathrm{S}$ de plantas daninhas têm sido feitos com plantas daninhas do tipo folha larga resistente aos herbicidas do grupo químico das s-triazinas. A maior parte desses estudos mostra prejuízo para o biótipo R em relação ao S (LeBaron \& Gressel, 1982). No entanto, estudos recentes têm evidenciado que biótipos de plantas daninhas resistentes a outros grupos químicos de herbicidas, como 
as sulfoniluréias e imidazolinonas (inibidores da ALS), não apresentam diferenças de adaptabilidade entre os biótipos R e S (Christoffoleti, 1992).

Murphy et al. (1986) avaliaram diversas características biológicas que caracterizam a adaptabilidade ecológica de populações resistente e suscetiveis da planta daninha Eleusine indica aos herbicidas do grupo químico das dinitroanilinas, em condições não-competitivas. Não foram encontradas diferenças significativas entre os biótipos em nenhuma das características estudadas, exceto para o peso da inflorescência. Gill et al. (1996) estudaram a germinação, o crescimento e o desenvolvimento de populações $\mathrm{R}$ e $\mathrm{S}$ de Lolium rigidum com mecanismos de resistência múltipla a várias classes de herbicidas. Não foi detectada nenhuma diferença significativa entre os biótipos com relação a dormência das sementes, taxa de crescimento relativo e fenologia.

O objetivo desta pesquisa foi comparar os biótipos de Bidens pilosa $\mathrm{R}$ e $\mathrm{S}$ aos herbicidas inibidores da ALS por meio da análise de crescimento de plantas individuais, observandose o acúmulo de biomassa seca, a taxa de crescimento absoluto (TCA), a taxa de crescimento relativo (TCR), o acúmulo de área foliar e a taxa de assimilação fotossintética líquida (AFL).

\section{MATERIAL E MÉTODOS}

\section{Fonte de Sementes Resistentes e Suscetíveis}

As sementes de Bidens pilosa $\mathrm{R}$ foram obtidas de uma área localizada no município de São Gabriel do Oeste, no Estado de Mato Grosso do Sul, em uma propriedade cultivada com a cultura da soja, na qual herbicidas inibidores da ALS tinham sido aplicados por pelo menos oito anos sucessivos. Nas safras 93/94 e 94/95, foram observadas falhas no controle do picão-preto por estes herbicidas em alguns talhões da propriedade. Na safra 95/96, foram coletadas sementes de um dos talhões onde ocorreram falhas de controle.

Sementes de B. pilosa também foram colhidas da horta comunitária localizada a $25 \mathrm{~km}$ de distância do local de coleta do biótipo R, na área urbana de São Gabriel do OesteMS. Além de nunca terem sido aplicados herbicidas inibidores da ALS, a área em questão era completamente isolada das áreas com suspeita de resistência.

A coleta das sementes no campo foi feita escolhendo-se cerca de 50 plantas sadias e com sementes maduras, num total de aproximadamente $100 \mathrm{~g}$ de sementes. Procurou-se coletar no mínimo 20 inflorescências de cada planta. As sementes foram acondicionadas em sacos de papel, para posterior transporte até o laboratório do Departamento de Produção Vegetal da ESALQ/USP, onde foram armazenadas em local seco e temperatura ambiente.

As sementes foram então plantadas em caixas plásticas de $0,15 \times 0,25 \times 0,05 \mathrm{~m}$, contendo substrato constituído de duas partes de solo e uma de terra vegetal, com resultados da análise granulométrica e física dispostos na Tabela 1. No estádio de duas folhas, as plantas de B. pilosa foram pulverizadas com o herbicida chlorimuron-ethyl na dose de 20 g i.a. ha-1. Algumas das plantas sobreviventes ao tratamento foram transplantadas para vasos com $5 \mathrm{~L}$ de capacidade, até a produção de sementes. Estas sementes foram usadas para a instalação do experimento de análise de crescimento do biótipo R.

As sementes do biótipo suscetível foram usadas diretamente da amostra coletada no campo. Para comprovar a suscetibilidade das plantas, foram semeadas amostras em caixas plásticas, da mesma forma descrita para o biótipo resistente, sendo as plantas pulverizadas com chlorimuron-ethyl 50 g p.c. ha- ${ }^{-1}$.

\section{Instalação do Experimento}

No dia 15/3/97, dez sementes de cada biótipo da planta daninha Bidens pilosa resistente e suscetivel aos herbicidas inibidores da ALS foram semeadas a $1 \mathrm{~cm}$ de profundidade em vasos de 0,12 m de diâmetro, contendo a mistura de solo anteriormente descrita, dispostos em casa de vegetação do Departamento de Produção Vegetal da ESALQ/USP. As plantas foram irrigadas diariamente e fertilizadas a cada 14 dias. Quatorze dias após o plantio, cada vaso foi desbastado, permanecendo uma planta por vaso para os biótipos $\mathrm{R}$ e S.

Além da área foliar de cada planta por vaso, determinou-se a biomassa seca da parte aérea

Planta Daninha, Viçosa-MG, v.19, n.1, p.75-83, 2001 
total da planta, incluindo ramos e folhas, após secagem em estufa a $70^{\circ} \mathrm{C}$ por $48 \mathrm{~h}$, até peso constante. Foram feitas colheitas a partir dos 14 dias após o plantio, em intervalos semanais, até os 91 dias; portanto, 12 épocas de colheita com quatro repetições cada uma, totalizando 48 vasos para cada biótipo.

\section{Análise dos resultados}

Os resultados de biomassa seca e área foliar foram analisados de acordo com a função de Richards, na forma de (Richards, 1959):

$$
\log _{\mathrm{e}}^{\mathrm{w}}=\log _{\mathrm{e}}^{\mathrm{A}}-\frac{1}{\mathrm{~N}} * \log _{\mathrm{e}}^{\left(1+\mathrm{e}^{\left(-\mathrm{K}^{*}(\text { Dias }-\mathrm{T})\right.}\right)}
$$

em que $\mathrm{N}$ governa o formato da curva e determina a posição do ponto de inflexão; A representa a assintota máxima no tempo infinito; $\mathrm{K}$ é a taxa ou constante em que A é atingido; $\mathrm{T}$ estima o tempo quando a curva atinge o ponto de inflexão; e W representa os valores de área foliar ou biomassa seca.

Os dados da biomassa seca de B. pilosa, representando o acúmulo diário, ajustado à função de Richards, derivaram a TCA. A TCR foi obtida a partir da TCA dividida pela biomassa total da planta, representando o acúmulo de biomassa por dia por grama de biomassa total. A TAL representa a quantidade de biomassa produzida pela planta em um dia, por $\mathrm{cm}^{2}$ de folha (Causton et al., 1978).

\section{RESULTADOS E DISCUSSÃO}

Causton et al. (1978) ressaltaram que existem vantagens associadas ao ajuste de funções matemáticas nos resultados de crescimento das plantas: a) sumariza os dados de forma conveniente; b) estima atributos de crescimento derivado dos resultados; c) calcula taxa de crescimento relativo diferenciado da função logaritmica; e d) fornece informações por meio de parâmetros da equação que apresentam significados biológicos.

Em uma função assintótica, o valor da variável dependente gradualmente ascende a um valor máximo que na realidade nunca é atingido, mas sim tangenciado. Há diversas funções assintóticas que têm sido utilizadas para análise de crescimento; no entanto, a função de Richards é a mais comumente usada. Ela é uma função logística desenvolvida por Richards (1959) que inclui as funções monomoleculares, Gompertz e logística como casos especiais (Hunt, 1982). Inicialmente, foi aplicado o procedimento PROC PLOT do programa de software estatístico SAS nos resultados médios da biomassa (gráfico não apresentado); com isso, foi possivel observar clara evidência de tendência de os resultados assumirem uma forma de curva assintótica.

Tabela 1 - Análises química e granulométrica de macro e alguns micronutrientes presentes na mistura de terra com material vegetal que foi usada nos experimentos

\begin{tabular}{|c|c|c|c|c|c|c|c|c|}
\hline \multirow{2}{*}{$\begin{array}{c}\mathrm{pH} \\
\mathrm{CaCl}_{2}\end{array}$} & \multirow{2}{*}{$\begin{array}{l}\text { M.O. } \\
\mathrm{g} \mathrm{dm}^{-3}\end{array}$} & $\mathrm{P}$ & $\mathrm{S}-\mathrm{SO}_{4}$ & $\mathrm{~K}$ & $\mathrm{Ca}$ & $\mathrm{Mg}$ & $\mathrm{Al}$ & \multirow{2}{*}{ SB } \\
\hline & & \multicolumn{2}{|c|}{----- $\mathrm{mg} \mathrm{dm}^{-3}$} & \multicolumn{4}{|c|}{ - } & \\
\hline 6,4 & 47 & 468 & 127 & 15,2 & 111 & 29 & 0 & 155,2 \\
\hline $\mathrm{T}$ & $\mathrm{V} \%$ & $\mathrm{~B}$ & \multicolumn{2}{|c|}{$\mathrm{Cu}$} & $\mathrm{Fe}$ & $\mathrm{Mn}$ & $\mathrm{Zn}$ & $\mathrm{Na}$ \\
\hline \multicolumn{9}{|c|}{ - } \\
\hline 170,2 & 91 & 1,51 & \multicolumn{2}{|c|}{6,1} & 30,8 & 29,0 & 15,0 & 402,50 \\
\hline
\end{tabular}

\begin{tabular}{|c|c|c|c|c|c|}
\hline \multicolumn{7}{|c|}{ Areia (\%) } \\
\hline Muito grossa & Grossa & Média & Fina & Muito Fina & Total \\
\hline 1 & 4 & 11 & 25 & 6 & 47 \\
\hline
\end{tabular}

\begin{tabular}{|c|c|c|c|}
\hline \multirow{2}{*}{ Silte (\%) } & \multicolumn{2}{|c|}{ Argila (\%) } & \multirow{2}{*}{ Classe de Textura } \\
\cline { 2 - 3 } & Total & Água & Argiloso \\
\hline 17 & 36 & 28 & An \\
\hline
\end{tabular}

- Resultados fornecidos pelo Laboratório de Análise de Solo do Departamento de Solos e Nutrição de Plantas - ESALQ/USP. 
Na Tabela 2 encontram-se listados os parâmetros da equação de Richards, obtidos a partir dos resultados de peso da biomassa dos biótipos R e S de B. pilosa; foi usado o procedimento estatístico não-linear do SAS, atribuindo-se valores arbitrários para A, K e T para que houvesse ajuste final da curva pelo programa. O valor de $\mathrm{F}$ (ajuste) representa a precisão de ajuste da função de Richards, chamado de "lack of fit". Ele é calculado por meio da divisão dos quadrados médios do erro da análise de variância geral dos resultados pelo quadrado médio dos erros da função de Richards (Garret et al., 1989). Os valores de F (ajuste) não-significativo indicam que o ajuste foi satisfatório e que a função de Richards representa de forma adequada os resultados de biomassa, não havendo diferenças significativas entre os erros dos dois modelos. Os coeficientes de correlação $\mathrm{R}^{2}$ comprovam também os ajustes adequados da função de Richards, pois a função explica mais de 90\% da variação dos resultados dos dois biótipos (Tabela 2).
A constante A estima a assintota máxima, ou seja, o máximo de crescimento que a planta de cada biótipo atinge (Garret et al., 1989). Como os valores de A são próximos dos valores máximos obtidos no experimento, evidenciase que a função de Richards foi um bom ajuste para ambos os biótipos. Os valores de A dos dois biótipos são bastante próximos - 125,01 (R) e 118,4 (S), e os intervalos de confiança de 95\% são coincidentes, provando que não existe diferença estatisticamente significativa entre as assintotas dos biótipos R e S (Tabela 2).

O parâmetro $\mathrm{N}$ define o formato da curva, localizando o ponto de inflexão, e pode variar de -1 até infinito. Todos os valores de $\mathrm{N}$ para os dois biótipos foram maiores que zero e bastante próximos de um, mostrando que os dois biótipos apresentam curvas de crescimento com ponto de inflexões similares. Essa conclusão é reafirmada pelos valores dos intervalos de confiança a 95\%, que são coincidentes (Tabela 1).

Tabela 2 - Parâmetros da função de Richards para as curvas de crescimento, derivada da biomassa seca (g) por planta de B. pilosa dos biótipos resistente e suscetível aos herbicidas inibidores da ALS, do experimento conduzido em casa de vegetação

\begin{tabular}{|c|c|c|c|c|c|c|c|}
\hline \multirow{2}{*}{ Parâmetros ${ }^{1}$} & \multirow{2}{*}{$\mathrm{R}$} & \multicolumn{2}{|c|}{ Inter. de conf. (95\%) } & \multirow{2}{*}{3} & \multirow{2}{*}{$\mathrm{S}$} & \multicolumn{2}{|c|}{ Inter. de conf. (95\%) } \\
\hline & & Inferior & Superior & & & Inferior & Superior \\
\hline $\mathrm{A}$ & 125,01 & 121,40 & 128,62 & n.s. & 118,14 & 114,08 & 122,20 \\
\hline $\mathrm{N}$ & 0,91 & 0,72 & 1,10 & n.s. & 0,96 & 0,56 & 1,37 \\
\hline $\mathrm{K}$ & 0,26 & 0,22 & 0,30 & n.s. & 0,19 & 0,14 & 0,24 \\
\hline $\mathrm{T}_{0}$ & 32,86 & 31,93 & 33,78 & n.s. & 29,70 & 26,85 & 32,56 \\
\hline TCA médio & 0,136 & 0,128 & 0,143 & $* *$ & 0,096 & 0,089 & 0,101 \\
\hline $\mathrm{R}^{2}$ & \multicolumn{3}{|c|}{0,91} & \multicolumn{4}{|c|}{0,95} \\
\hline $\mathrm{F}($ modelo $)$ & \multicolumn{3}{|c|}{$1924,19 * *$} & \multicolumn{4}{|c|}{$501,73 * *$} \\
\hline $\mathrm{F}(\text { ajuste })^{2}$ & \multicolumn{3}{|c|}{0,44 n.s. } & \multicolumn{4}{|c|}{0,48 n.s. } \\
\hline
\end{tabular}

1 Dados da biomassa seca dos biótipos R e S de B. pilosa foram ajustados à função de Richards (Richards, 1959), em que w é a biomassa em função do tempo; A é o valor assintótico de w; $\mathrm{N}$ governa o formato da curva e determina a posição do ponto de inflexão; A representa a assintota máxima no tempo infinito; $\mathrm{K}$ é a taxa ou constante em que A é atingido; e $\mathrm{T}$ estima o tempo quando a curva atinge o ponto de inflexão.

2 F que mede a precisão do ajuste da regressão não-linear (função de Richards) em relação à análise de variância do modelo geral.

3 Parâmetros da função de Richards dos biótipos R e S não diferem estatisticamente entre si (n.s. = não-significativo, pois os intervalos de confiança dos dois biótipos são coincidentes).

n.s. = não-significativo estatisticamente; $* *$ significativo estatisticamente a $1 \%$.

$\mathrm{R}^{2}=$ coeficiente de correlação do modelo não-linear (função de Richards). 
O parâmetro $\mathrm{T}_{0}$ é a estimativa de quando a curva atinge o ponto de inflexão (Garret et al., 1989). Os resultados constantes na Tabela 2 evidenciam que os parâmetros $\mathrm{K}$ e $\mathrm{T}_{0}$ são estatisticamente semelhantes para ambos os biótipos, pois os intervalos de confiança a 95\% são coincidentes. $\mathrm{O}$ valor de $\mathrm{K}$ em si não tem muito significado biológico. Ele pode ser interpretado com uma medida da taxa em que A é atingido e pode ser usado para calcular a taxa de crescimento absoluto, por meio da fórmula $\mathrm{K} /(\mathrm{N}+1)$.

A função de Richards ajustada aos dados de biomassa seca, transformados para logaritmo natural através do exponencial dos biótipos R e S, está representada na Figura 1. Comparando os resultados obtidos na Figura $1 \mathrm{com}$ os valores dos intervalos de confiança presentes na Tabela 3, observa-se que o biótipo S acumulou mais biomassa até a terceira avaliação, quando comparado com o biótipo R, porém a partir daí o biótipo R igualou-se em peso da biomassa.

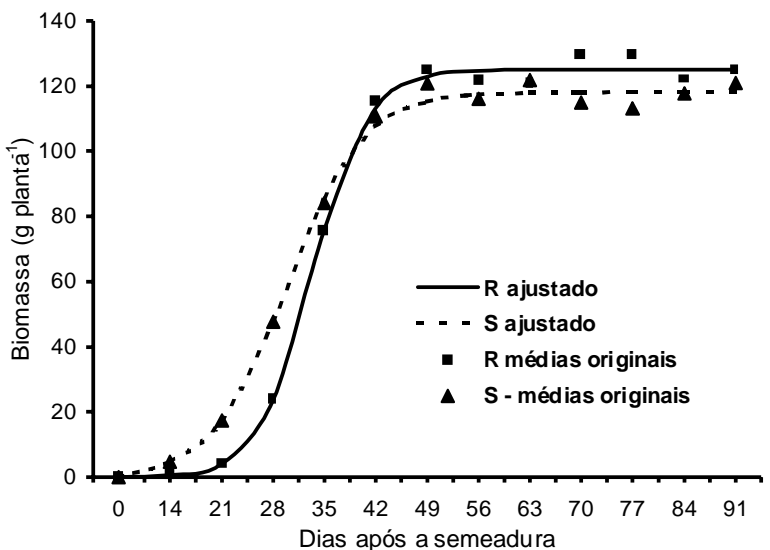

Figura 1 - Valores de biomassa calculados através da função de Richards (linhas) e das médias (símbolos), derivados do peso da biomassa por planta de B. pilosa dos biótipos resistente e suscetível aos herbicidas inibidores da ALS, obtidos em casa de vegetação.

Tabela 3 - Peso da biomassa da parte aérea por planta de B. pilosa dos biótipos resistente e suscetível aos herbicidas inibidores da ALS, medido semanalmente a partir dos 14 dias após a semeadura, e limites superior e inferior dos intervalos de confiança de $95 \%$

\begin{tabular}{|c|c|c|c|c|c|c|c|}
\hline \multirow{3}{*}{ DAS } & \multicolumn{7}{|c|}{ Biótipos } \\
\hline & \multicolumn{3}{|c|}{ Resistente } & \multicolumn{4}{|c|}{ Suscetível $^{1}$} \\
\hline & inferior $^{1}$ & biomassa $^{2}$ & superior $^{1}$ & & inferior $^{1}$ & biomassa $^{2}$ & superior $^{1}$ \\
\hline 14 & 0,52 & 0,58 & 0,67 & $*$ & 3,24 & 4,78 & 5,50 \\
\hline 21 & 3,94 & 4,06 & 4,67 & $*$ & 13,31 & 17,18 & 19,76 \\
\hline 28 & 22,52 & 23,81 & 27,38 & $*$ & 37,76 & 47,69 & 54,84 \\
\hline 35 & 67,92 & 75,96 & 79,89 & n.s. & 69,88 & 85,80 & 88,57 \\
\hline 42 & 104,46 & 113,32 & 119,63 & n.s. & 93,71 & 107,69 & 113,95 \\
\hline 49 & 117,28 & 122,95 & 127,46 & n.s. & 105,83 & 115,25 & 120,60 \\
\hline 56 & 120,47 & 124,67 & 128,48 & n.s. & 110,92 & 117,38 & 121,91 \\
\hline 63 & 121,20 & 124,67 & 128,60 & n.s. & 112,90 & 117,94 & 122,15 \\
\hline 70 & 121,36 & 124,95 & 128,62 & n.s. & 113,65 & 118,09 & 122,19 \\
\hline 77 & 121,39 & 125,00 & 128,62 & n.s. & 113,92 & 118,13 & 122,20 \\
\hline 84 & 121,40 & 125,01 & 128,62 & n.s. & 114,02 & 118,14 & 122,20 \\
\hline 91 & 121,40 & 125,01 & 128,62 & n.s. & 114,06 & 118,14 & 122,20 \\
\hline
\end{tabular}

DAS $=$ dias após semeadura .

1 Limites inferior e superior de intervalo de confiança de 95\% de B. pilosa de biótipos resistente e suscetível aos herbicidas inibidores da ALS, extraídos da função de Richards.

2 Resultados de biomassa seca para os biótipos resistente e suscetível de B. pilosa, calculada através da função de Richards.

- Asteriscos indicam diferença significativa entre os biótipos (probabilidade p<0,05); n.s. = não-significativo, baseado no intervalo de confiança de $95 \%$. 
A TCA média dá uma estimativa do desempenho geral da planta durante todo o período de crescimento estudado, mostrando que o biótipo $\mathrm{R}$ foi superior ao biótipo $\mathrm{S}$, pois 0,136 foi estatisticamente superior a 0,096 (Tabela 2). No entanto, na Figura 2, observa-se que no período inicial de desenvolvimento da planta o biótipo R teve TCA menor que S, superando-o a partir dos 35 dias após semeadura. Esses resultados explicam por que o biótipo $\mathrm{R}$ apresentou plantas de menor tamanho no início de seu desenvolvimento, na fase de plântula, porém no final tinha tamanho semelhante ao biótipo S. A taxa máxima de crescimento do biótipo $\mathrm{R}$ foi em torno de $7,8 \mathrm{~g} \mathrm{dia}^{-1}$, e a do biótipo $\mathrm{S}$, de $5,7 \mathrm{~g} \mathrm{dia}^{-1}$.

Os resultados da Tabela 4 confirmam as tendências observadas na Figura 2, pois as diferenças entre as TCAs dos biótipos são significativamente maiores para o biótipo $\mathrm{S}$ até os 28 dias após semeadura, porém a partir dos 28 até os 42 dias após a semeadura o biótipo $\mathrm{R}$ apresentou TCA significativamente maior.
Essas diferenças podem ser observadas comparando os intervalos de confiança a 95\% dos dois biótipos. A partir dos 49 dias após a semeadura, os biótipos não apresentaram diferenças estatisticamente significativas entre si com relação à TCA.

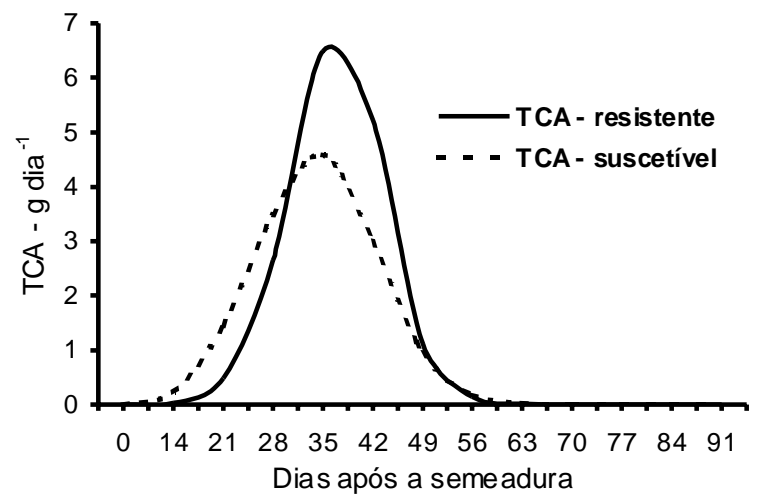

Figura 2 - Taxa de crescimento absoluto (TCA) derivado da função de Richards a partir dos valores de peso da biomassa por planta dos biótipos resistentes (R) e suscetíveis (S) de B. pilosa, do experimento conduzido em casa de vegetação.

Tabela 4 - Taxa de crescimento absoluto derivado da função de Richards a partir dos valores da biomassa da parte aérea por planta dos biótipos R e S de B. pilosa e limites superior e inferior dos intervalos de confiança de $95 \%$

\begin{tabular}{|c|c|c|c|c|c|c|c|}
\hline \multirow{3}{*}{ DAS } & \multicolumn{7}{|c|}{ Biótipos } \\
\hline & \multicolumn{4}{|c|}{ Resistente } & \multicolumn{3}{|c|}{ Suscetível } \\
\hline & inferior $^{1}$ & biomassa $^{2}$ & superior $^{1}$ & & inferior $^{1}$ & biomassa $^{2}$ & superior $^{1}$ \\
\hline 14 & 0,04 & 0,03 & 0,05 & $*$ & 0,34 & 0,23 & 0,36 \\
\hline 21 & 0,50 & 0,49 & 0,51 & * & 1,77 & 1,44 & 1,89 \\
\hline 28 & 2,71 & 2,65 & 2,82 & * & 4,36 & 3,49 & 4,45 \\
\hline 35 & 7,45 & 6,49 & 8,15 & $*$ & 5,44 & 4,59 & 6,37 \\
\hline 42 & 5,34 & 5,22 & 5,68 & * & 3,13 & 3,00 & 3,62 \\
\hline 49 & 1,38 & 1,12 & 1,83 & n.s. & 1,08 & 0,95 & 1,73 \\
\hline 56 & 0,25 & 0,15 & 0,46 & n.s. & 0,30 & 0,19 & 0,73 \\
\hline 63 & 0,04 & 0,02 & 0,10 & n.s. & 0,08 & 0,03 & 0,28 \\
\hline 70 & 0,006 & 0,002 & 0,023 & n.s. & 0,02 & 0,006 & 0,10 \\
\hline 77 & 0,001 & 0,0003 & 0,005 & n.s. & 0,005 & 0,001 & 0,04 \\
\hline 84 & 0,0002 & 0,0001 & 0,001 & n.s. & 0,001 & 0,0002 & 0,014 \\
\hline 91 & 0,0001 & 0,00001 & 0,0002 & n.s. & 0,0004 & 0,00001 & 0,005 \\
\hline
\end{tabular}

DAS = dias após semeadura.

1 Limites inferior e superior de intervalo de confiança de $95 \%$ de B. pilosa de biótipos resistente e suscetível aos herbicidas inibidores da ALS, extraídos da função de Richards.

2 Resultados de TCA para os biótipos resistente e suscetível de B. pilosa, calculada através da função de Richards.

- Asteriscos indicam diferença significativa entre os biótipos (probabilidade $\mathrm{p}<0,05$ ); n.s. = não-significativo, baseado no intervalo de confiança de $95 \%$. 
Esses resultados podem explicar por que o biótipo $\mathrm{R}$ é mantido em baixa freqüência na ausência do herbicida como agente de pressão de seleção. Guando o herbicida não é aplicado, a TCA do biótipo S impede que o biótipo R ocupe nicho ecológico; no entanto, quando o herbicida é aplicado, há ausência do biótipo S, e, portanto, o biótipo R desenvolve-se, mesmo com menor TCA que o suscetivel.

A TCR é considerada um índice de eficiência da planta, pois ela reflete a capacidade produtiva diária de biomassa produzida por grama de biomassa da planta. Os resultados indicam que a taxa de crescimento relativo, TCR (dados não apresentados) da planta foi semelhante nos primeiros dias de desenvolvimento; entretanto, durante a fase intermediária de crescimento, o biótipo $\mathrm{R}$ foi mais eficiente que o $\mathrm{S}$. No final, ambos os biótipos apresentavam a mesma TCR. Assim, neste experimento, a maior eficiência do biótipo $\mathrm{R}$ compensou seu menor tamanho inicial, de forma que no final ambos os biótipos apresentavam mesmo tamanho.

Os acúmulos de área foliar foram também avaliados pela função de Richards. Normalmente, o ajuste desta função não é muito adequado para a área foliar, pois esta normalmente decai no final do ciclo, e, assim, os valores das últimas avaliações não são assintóticos a um valor máximo. No entanto, no caso dos biótipos $\mathrm{R}$ e S estudados nesta pesquisa, a última coleta do valor de área foliar foi antes do início da queda no valor da área foliar. Pelos resultados contidos na Figura 3, verifica-se que os dois biótipos tiveram valores bem próximos, porém novamente a tendência observada para o acúmulo de biomassa repetiu-se para a área foliar, ou seja, no início do desenvolvimento, a planta S era mais folhosa que a planta $\mathrm{R}$.

Um parâmetro composto da combinação de duas taxas (acúmulo de biomassa e área foliar) é a taxa de assimilação fotossintética líquida (TAL), a qual mede a eficiência da folha na produção de biomassa, isto é, a quantidade em gramas de biomassa produzida por $\mathrm{cm}^{2} \mathrm{de}$ folha por dia. A TAL é o parâmetro composto mais representativo da eficiência de uma planta, pois ele é um índice de crescimento da planta, independentemente de seu tamanho. Novamente, o biótipo S apresenta eficiência maior que o biótipo R no início do ciclo da planta daninha, até os 30 DAS (Figura 4).

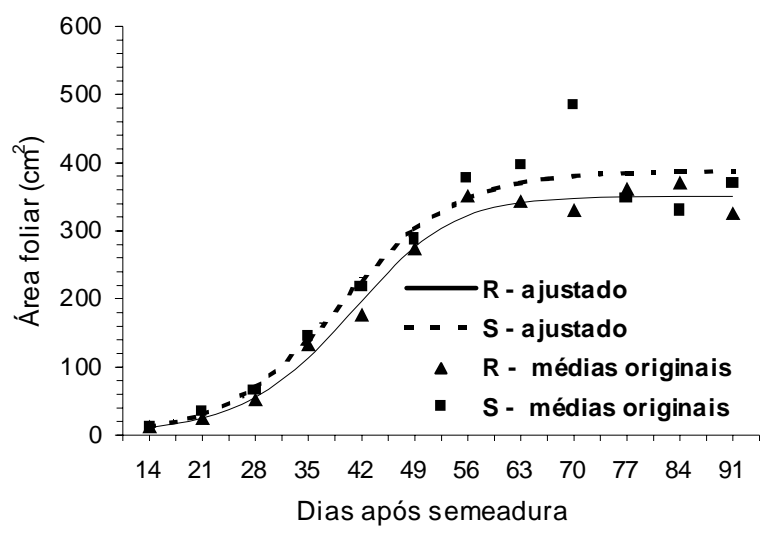

Figura 3 - Valores de área foliar calculados através da função de Richards (linhas) e valores médios das quatro repetições (símbolos) derivados da área foliar por planta dos biótipos resistente e suscetível de $B$. pilosa aos herbicidas inibidores da ALS, do experimento conduzido em casa de vegetação.

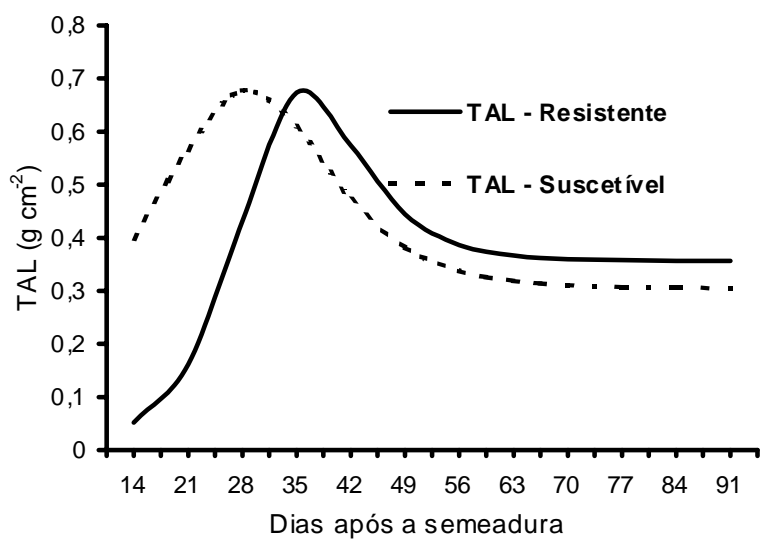

Figura 4 - Taxa de assimilação líquida (TAL) derivada da função de Richards a partir dos valores de peso da biomassa e área foliar por planta dos biótipos resistentes $(\mathrm{R})$ e suscetíveis (S) de B. pilosa, do experimento conduzido em casa de vegetação.

Dentre as conclusões gerais deste trabalho pode-se inferir que não existe diferença evidente nos padrões de crescimento entre os biótipos de $B$. pilosa $\mathrm{R}$ e $\mathrm{S}$ aos herbicidas inibidores da ALS sob condições não-competitivas. Contrariamente ao que acontece com alguns biótipos de plantas daninhas resistentes aos herbicidas inibidores da fotossíntese, os resultados desta pesquisa demonstram que a modificação causada na ALS, que confere resistência, não está correlacionada a decréscimo de produtividade da planta. A semelhança na eficiência de produção de biomassa e área foliar dos biótipos R e S de Bidens pilosa indica que ambos 
apresentam mesma adaptabilidade ecológica e ocupam nichos semelhantes no ambiente. Dessa forma, estratégias preventivas de resistência devem ser adotadas, pois, uma vez estabelecida uma população resistente, naturalmente ela não retorna para uma freqüência original de suscetibilidade.

\section{LITERATURA CITADA}

CAUSTON, D.R.; EliAS, C.O.; HADlEY, P. Biometrical studies of plant growth. I. The Richards function, and its application in analyzing the effects of temperature on leaf growth. Plant Cell Environ., v.1, p.163-184, 1978.

CHRISTOFFOLETI, P.J. Growth, competitive ability and fitness of sulfonylurea resistant and susceptible Kochia scoparia. Fort Collins: Colorado State University, 1992. 198p.

DYER, W.E.; CHEE, P.W.; FAY, P.K. Rapid germination of sulfonylurea-resistant Kochia scoparia accessions is associated with elevated seed levels of branched chain amino acids. Weed Sci., v.41, p.18-22, 1993.

FRANCE, J.; THORNLEY, H.M. Mathematical models in agriculture. Kent Butterworths \& Co., 1984. 335p.

GARRET, G.A.; McSAY; MOORE III, F. RF, a computer program to fit a generalization of the logistic function using D.R. Causton and J.C. Venus methods. Fort Collins: Colorado State University, 1989. p.89-104.

GILL, G.S.; COUSENS, R.D; ALLAN, M.R. Germination, growth, and development of herbicide resistant and susceptible populations of Rigid Ryegrass (Lolium rigidum). Weed Sci., v.44, p.252-256, 1996.
GRESSEL, J.; SEGEL, L.A. The paucity of plants evolving genetic resistance to herbicides: possible reasons and implications. J . Theoric. Biol., v.75, p.349-371, 1978.

HUNT, R. Plant growth curves - the functional approach to plant growth analysis. Sheffield: Edward Arnold, Publ., 1982. 248p.

LeBARON, H.M.; GRESSEL, J. Summary of accomplishments, conclusions, and future needs. In: LeBARON, H.; GRESSEL, J. (Ed.) Herbicide resistance in plants. New York: John Wiley and Sons, 1982. p.349-362.

MAPPLEBECK, L.R.; SOUZA MACHADO, R.; GRODZINSKI, B. Seed germination and seedlings growth characteristics of atrazinesusceptible and resistant biotypes of Brassica campestris. Can. J . Plant Sci., v.62, p.733-739, 1982.

MAXWELL, B.D.; ROUSH, M.L.; RADOSEVISH, R. Predicting the evolution and dynamics of herbicide resistance in weed populations. Weed Technol., v.4, p.2-13, 1990.

MURPHY, T.R.; GOSSETT, B.J.; TOLER, J.E. Growth and development of dinitroanilinesusceptible and -resistant goosegrass (Eleusine indica) biotypes under noncompetitive conditions. Weed Sci., v.34, p.704-710, 1986.

RICHARDS, F.J. A flexible growth function for empirical use. J . Exper. Bot. , v.10, p.290-300, 1959.

THOMPSON, C.R.; THILL, D.C.; SHAFI. Germination characteristics of sulfonylurearesistant and -susceptible kochia (Kochia scoparia). Weed Sci., v.4, p.50-56, 1996. 